Relations industrielles

Industrial Relations

\title{
La part des salaires dans le revenu national au Canada, 1910 à 1980
}

\section{The Wage Share in Canada, 1910-1980}

\section{Gérald Marion}

\section{Volume 37, numéro 1, 1982}

URI : https://id.erudit.org/iderudit/029232ar

DOI : https://doi.org/10.7202/029232ar

Aller au sommaire du numéro

Éditeur(s)

Département des relations industrielles de l'Université Laval

ISSN

0034-379X (imprimé)

1703-8138 (numérique)

Découvrir la revue

Citer cet article

Marion, G. (1982). La part des salaires dans le revenu national au Canada, 1910 à 1980. Relations industrielles / Industrial Relations, 37(1), 53-64.

https://doi.org/10.7202/029232ar
Résumé de l'article

De 1910 à 1980, la part salariale dans le revenu national a augmenté de trente (30) points en pourcentage. Cette hausse s'accompagne d'une augmentation de la proportion de salariés dans la population ainsi que de changements de structure de l'économie. C'est le but de cet article de dégager les grandes tendances de la part salariale ainsi que de quantifier l'importance des facteurs qui les sous-tendent.
Tous droits réservés (C) Département des relations industrielles de l'Université Laval, 1982
Ce document est protégé par la loi sur le droit d'auteur. L'utilisation des services d'Érudit (y compris la reproduction) est assujettie à sa politique d'utilisation que vous pouvez consulter en ligne.

https://apropos.erudit.org/fr/usagers/politique-dutilisation/ 


\section{La part des salaires dans le revenu national au Canada, 1910 à 1980}

\section{Gérald Marion}

De 1910 à 1980, la part salariale dans le revenu national a augmenté de trente (30) points en pourcentage. Cette hausse s'accompagne d'une augmentation de la proportion de salariés dans la population ainsi que de changements de structure de l'économie. C'est le but de cet article de dégager les grandes tendances de la part salariale ainsi que de quantifier l'importance des facteurs qui les sous-tendent.

La part des salaires dans le revenu a toujours fait l'objet des préoccupations des spécialistes, surtout depuis que la théorie keynésienne a ravivé l'intérêt pour les études sur la demande globale. Au Canada, nous possédons des informations sur la masse salariale qui remontent au début du siècle. Comme l'évolution de ces grands agrégats se fait lentement sur des périodes séculaires, il nous est maintenant possible de procéder à des comparaisons historiques de plus en plus révélatrices. Nous avons à diverses occasions étudié l'évolution de la part salariale pour des périodes plus ou moins longues. Notre but maintenant est d'en dégager les grandes tendances durant ce dernier trois quarts de siècle.

On sait qu'il existe plusieurs concepts du revenu national. Pour les fins de la présente étude, il est préférable de s'en tenir au «revenu national au coût des facteurs». Ce concept reflète la dimension coût du processus de production. Mais, comme la concurrence est généralement moins prononcée sur le marché des facteurs que sur celui des produits finis, on peut objecter que ce concept ne mesure pas aussi bien le bien-être de la population que le produit national au prix du marché, lié au marché de la consommation finale. Néanmoins, la pureté du concept «au prix des facteurs» nous incline à le choisir comme valeur référence pour mesurer la part salariale. En effet, cet agrégat n'inclut que la rémunération des facteurs de production. Les réserves pour amortissements de même que certains ajustements en vue d'éli-

* MARION, Gérald, professeur, Faculté des arts et des sciences, Université de Montréal.

Relat. ind., vol. 37, no 1 (1982) () PUL ISSN 0034-379 X 
miner les dépenses intermédiaires des gouvernements n'ayant pas à y être pratiqués, il nous paraît moins sujet à des erreurs ou à des fluctuations arbitraires que le concept «au prix du marché», et ainsi plus approprié pour évaluer la part salariale.

Quant à la masse salariale, elle inclut, en plus des salaires et traitements, le salaire des militaires. La précision de cet agrégat s'est amélioré depuis le début du siècle, mais il demeure insatisfaisant sous certains rapports. D'abord, la rémunération du travail n'est pas complète puisqu'il n'y a pas d'imputation pour le travail des aides-familiaux non rémunérés. Comme l'importance de ce facteur a diminué sur la période étudiée il y a une source de biais que nous tenterons d'évaluer. De plus, cet agrégat inclut tout le revenu des salariés, y compris celui des administrateurs ce qui, dans le contexte des changements institutionnels qui se sont produits avec l'incorporation des petites entreprises, a pour effet d'en accroître la masse.

\section{TENDANCE, 1910-1978}

Nous possédons sur le revenu national des statistiques qui remontent à 1890. Mais pour la masse salariale nous ne pouvons remonter qu'à $1910^{1}$, date qui marque le début de l'étude.

La moyenne 1900-1911 du revenu national au coût des facteurs se situe à un peu plus de deux milliards de dollars, alors que la masse salariale atteint 907 millions. La part des salaires dans le revenu national au coût des facteurs se situe donc à $43 \%$. Par la suite, cette part salariale allait connaître, à long terme, une hausse de plus de 30 points pour atteindre $74 \%$ du revenu national dans les années ' 70 . Cette évolution cependant a été loin d'être régulière: les fluctuations cycliques, celles des années ' 30 notamment, l'emportent parfois sur la variation à long terme ${ }^{2}$.

De façon détaillée, la première augmentation importante se situe d'abord entre 1910 et 1920 . Au sommet de la conjoncture en 1920, le revenu national atteint quatre milliards et demi de dollars et la masse salariale deux milliards et demi. La part salariale connaît donc une hausse de 11 points passant à $55 \%$ du revenu national. Contrairement au modèle qui allait se

1 En fait, il nous a été possible, à partir de diverses sources, d'établir des séries cohérentes jusqu'à cette date.

2 Ces conclusions ainsi que celles qui suivent reposent sur des études plus techniques que nous avons faites. Voir, Gérald MARION, Répartition fonctionnelle des revenus: Analyse de la part du travail au Canada, P.U.M., 1965; G. MARION et Aly BOURY SY, La part salariale dans le revenu national: L'expérience contemporaine, Cahier 8114, Département de sciences économiques, Université de Montréal, 1981. 
développer à partir des années ' 30 , il ne se produit pas de hausse de la part salariale durant la chute rapide des prix de 1921: le revenu national et la masse salariale baissent dans des proportions semblables de sorte que la part des salaires se maintient à $55 \%$.

Durant les années de prospérité qui caractérisent la décennie des années '20, il semble se produire une légère hausse de la part salariale, quoique la faible variation, environ deux points, de pair avec la fragilité des statistiques à cette époque, ne nous permet pas de conclusions fermes. Par la suite, les années de dépression avec la volatilisation des profits et la réduction de la production nationale qui baisse à 2.3 milliards en 1933, voient la part salariale monter à $75 \%$ du revenu national. Il ne s'agit bien sûr que d'une hausse conjoncturelle, c'est-à-dire de variations contracycliques de la part salariale qui d'ailleurs vont, à partir de 1930, caractériser la période que nous étudions. En effet, contrairement à ce que nous avons observé durant les années '20, les années de dépression de 1930-1933 voit la part salariale augmenter alors qu'elle baisse à $63 \%$ avec la reprise de la fin des années ' 30 .

Durant les années de contrôle de guerre elle continue de se maintenir autour de ce niveau. Ce qui, vu dans le long terme, représente une hausse de sept points par rapport à son niveau atteint en 1920.

Ce n'est qu'au milieu de la décennie 1950 que la part salariale allait atteindre de nouveaux paliers passant d'abord à $64 \%$, puis, suivant le modèle contracyclique, à $68 \%$ lors de la récession de 1957 , pour atteindre, après de légères fluctuations à la baisse, $70 \%$ en 1965 . Il s'agit donc pour la décennie 1955-1965, d'une hausse de 10 points. Enfin, la dernière décennie, débutant en 1970, a vu la part salariale dépasser résolument le seuil de $70 \%$ pour osciller autour de $73 \%$. Ainsi, pour l'ensemble de la période, la part salariale gagne 30 points sur la base des $43 \%$ qu'elle obtenait en 1910 .

\section{FLUCTUATIONS CYCLIQUES DE LA PART SALARIALE}

Avant de passer à l'étude de la tendance à long terme, nous analysons brièvement les fluctuations cycliques de la part salariale.

Depuis 1930, la baisse de l'activité économique conduit à la hausse de la part du produit attribué aux salariés alors que l'inverse se produit en période de reprise économique. Par ailleurs, sauf durant les années récentes où les anticipations d'inflation ont perturbé la relation salaire-chômage, on a observé, notamment pour la période d'après-guerre, une relation inverse entre le taux d'augmentation des salaires nominaux et les taux de variation du chômage. Pourtant, comme nous venons de le mentionner, en ce qui con- 
cerne la part salariale, depuis 1930, c'est généralement l'inverse que l'on observe. Ce qui signifie que ce ne sont pas les salaires nominaux qui poussent à des variations contracycliques de la part salariale. Reste donc l'emploi. Cette variable, qui avec les salaires nominaux déterminent la masse salariale et en conséquence les mouvements de la part salariale, devrait être le facteur qui empêche la masse salariale de baisser autant que la production. En d'autres mots en période de ralentissement de l'économie la masse salariale décroît moins vite que la production, ce qui ne paraît pas être dû aux mouvements salariaux, qui selon la théorie de la courbe de Phillips ralentissent également en période de récession. L'explication de la hausse de la part salariale repose alors sur l'emploi, c'est-à-dire sur les décalages de l'ernploi par rapport à la production. Ainsi, si l'emploi est rigide quand la production baisse, la masse salariale (emploi et salaire) aura tendance à décroître moins que le produit national. Dès lors la part salariale augmentera ${ }^{3}$.

En fait, nous observons des délais de l'ajustement de l'emploi à la production. En d'autres mots, la pratique, en période de ralentissement économique, de retarder le licenciement de la main-d'oeuvre ou de retenir les travailleurs qualifiés afin d'éviter les coûts de réembauche et de réentraînement de ceux-ci lors de la reprise conjoncturelle, fait que l'emploi tarde à s'ajuster à des diminutions de production. L'inverse se produisant en période de reprise. Ces pratiques amènent des mouvements contracycliques de la part salariale.

Durant la période de la grande dépression, sans aucun doute, la volatilisation des profits est la première cause de la hausse de la part salariale, mais durant l'après-guerre, l'écart entre l'emploi désiré dû aux délais de licenciement de la main-d'oeuvre paraît en être une cause importante de ces mouvements contracycliques. Ainsi, durant la récession 1957-1958, alors que la part salariale hausse de plus de deux points pour atteindre un nouveau palier, l'emploi effectif est de $3 \%$ supérieur à l'emploi d'équilibre ${ }^{4}$.

3 La part salariale peut être représentée comme le produit des salaires réels et de la réci-

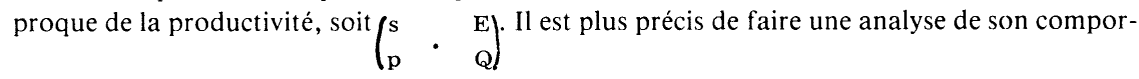
tement cyclique à partir des variations cycliques des salaires réels. Sur ce point voir J.M. KEYNES, La Théorie générale de l'emploi, de l'intérêt et de la monnaie, Payot, p. 10; Jacques RUEFF, «Nouvelle discussion sur le chômage, les salaires et les prix», Revue d'économie politique, p. 61, pp. 761-781, 1951. Voir également N. PARASIADES, Essai sur les relations entre le chômage, le salaire, les prix et le profit - Étude critique de la loi de M. Rueff, P.U.F., 1949. K. TARSHIS, "Changes in Real and Money Wages", Economic Journal, 48, sept. 1938; R.O. BODKIN, "Real Wages and Cyclical Variations in Employment. An Examination of the Evidence", Canadian Journal of Economics, II, no 3, août 1969.

4 MARION, Gérald, «Fonction d'emploi, taux de participation de la main-d'oeuvre et demande excédentaire de travail», Revue économique, vol. XX, no 6, novembre 1969. Pour une étude systématique de la relation entre la part salariale et la réserve de travail pour la période d'après-guerre, voir G. MARION et B.G. SPENCER, "Labour Hoarding and the Wage Share: Test of a Hypothesis", Relations industrielles, vol. 34, no 1, 1979. 
Cependant, nous n'avons pas observé ce phénomène avant $1930 \mathrm{au}$ Canada. Durant les années '20 la tendance est plutôt que la part salariale suit l'évolution de la conjoncture. Il semble que des pratiques industrielles différentes, en particulier une demande de main-d'oeuvre qualifiée moins élevée, qui atténue l'aspect permanent de l'emploi, expliquent cette allure différente de la part salariale au début de la période. Phelps-Brown qui a étudié le comportement de la part salariale dans divers pays occidentaux observe d'ailleurs que le phénomène de contracyclicité de la part salariale n'est apparu qu'au $\mathrm{XX}^{\mathrm{e}}$ siècle. $\mathrm{Au} \mathrm{XIX}^{\mathrm{e}}$ siècle, la part salariale évoluait suivant le cycle économiques.

\section{LA PART SALARIALE ET LE TAUX DE SALARIAT}

Il existe plusieurs façons d'aborder l'étude de l'évolution de la part salariale dans le long terme. La première et la plus manifeste repose sur l'évolution du taux de salariat, c'est-à-dire sur les variations de la proportion de la population active qui est salariée. Si les propriétaires de petites entreprises non incorporées liquident ou vendent leurs actifs pour devenir salariés, la proportion de salariés dans la population active s'en trouve accrue de même que la part salariale. En second lieu, nous pouvons chercher des explications de la hausse de la part salariale dans la structure de l'économie. On sait en effet que la proportion des revenus attribués aux salariés n'est pas la même dans tous les secteurs industriels ou commerciaux. Que les secteurs où cette proportion est élevée accroissent leur importance et la part salariale totale s'en trouvera accrue. Enfin, la part salariale dépend du taux de rémunération et du nombre de salariés d'une part, et du taux de rendement et du stock de capital, d'autre part.

Le taux de salariat, comme nous venons de le mentionner, est le rapport du nombre de salariés à la population active. Mais une question préalable se pose, car il existe plusieurs façons de définir les salariés. Doit-on y inclure les aides-familiaux ou mêmes les hauts dirigeants salariés des entreprises? Les aides-familiaux ayant connu une évolution importante, nous évaluons le taux de salariat à la fois en les incluant et en les excluant de l'agrégat.

D'abord disons que quels que soient les concepts que l'on retienne pour sa mesure, le taux du salariat a connu une hausse importante au Canada durant la période étudiée. Ainsi l'évalue-t-on en ne tenant compte que de ceux ayant un emploi, ce qui exclut aussi bien les chômeurs que les aides-

5 BROWN, Phelps et P.E. HART, "The Share of Wages in National Income", The Economic Journal, juin 1952. 
familiaux, le taux de salariat passe de $61 \%$ en 1921 à près de $90 \%$ en 1978 . Si par contre on tient compte des aides-familiaux, mais toujours sans les chômeurs, la hausse est toujours significative mais réduite par rapport à la première estimation, car les aides-familiaux sont surtout importants au début de la période. Dans ce cas, le taux de salariat se chiffre à $70 \%$ en 1921 et à un minimum de $90 \%$ en $1978^{6}$. Il demeure toujours une hausse de plus de 20 points en pourcentage?

Cette augmentation du taux de salariat signifie que des travailleurs indépendants changent de statut pour devenir salariés. En fait, dans cette optique, les «indépendants», professionnels ou propriétaires d'entreprises, sont la plaque tournante où s'effectuent les gonflements de la masse salariale. En d'autres mots, leur importance découle de la répartition originelle des revenus en trois catégories de facteurs: les salariés, les indépendants et le capital. Or, la période que nous étudions est marquée par une augmentation relative du nombre de sociétés à capitaux. Ce qui signifie que des petits propriétaires deviennent des salariés dans ces sociétés. La masse des revenus des indépendants diminue alors pour augmenter, en retour, la masse des salaires. En d'autres termes, au fur et à mesure que les indépendants deviennent des salariés, les facteurs primaires, travail et capital, qui se retrouvent simultanément chez les indépendants, rentrent dans leur catégorie respective de travail et capital. C'est un phénomène structurel qui a tendance à prendre place surtout en longue période, mais en courte période, les changements de prix relatifs peuvent également se traduire par de telles mutations.

Ainsi, lorsqu'il se produit une augmentation du nombre de salariés par la transformation des entreprises non-incorporées en société à capitaux il y a parallèlement, même en l'absence de changement des prix relatifs, une hausse relative de la masse salariale. Si le taux de salariat et la masse des salaires suivent un cheminement parallèle, la hausse de la part salarialle ne fait que refléter celle de l'augmentation du nombre de salariés par rapport aux non-salariés. Dans ce cas, le revenu moyen des salariés évolue au même rythme que celui des non-salariés. En effet, le rapport de la part salariale et du taux de salariat est égal au revenu relatif des salariés, c'est-à-dire au rapport des revenus des salariés à celui des non-salariés. Si le rapport de la part salariale au taux de salariat est stable, tel est aussi le cas du revenu moyen des salariés par rapport à celui des non-salariés.

6 Il s'agit d'un minimum, car nous retenons l'hypothèse que le nombre d'aidesfamiliaux non-rémunérés tend vers zéro.

7 Ces deux estimés représentent les deux extrêmes de la fourchette des calculs possibles. Les jeux de calcul que l'on peut faire avec l'inclusion des chômeurs s'y situe, en longue période, à l'intérieur. 
Qu'en est-il, en fait, de l'évolution de ce rapport? Si l'on compare l'évolution de la part salariale entre 1921 et 1978 avec celle du taux de salariat, n'incluant pas les aides-familiaux, on constate que la hausse du taux de salariat est plus élevée. Le revenu moyen des salariés baisse par rapport aux non-salariés. Ce qui est visible à la colonne (3) du tableau 1. En effet, en 1921 , le revenu moyen des salariés est égal à $90 \%$ de celui des non-salariés, travailleurs indépendants, alors qu'en 1978 , il est de $82 \%$ de celui des nonsalariés. Par ailleurs, si on inclut les aides-familiaux dans le calcul du taux de salariat, comme ce taux se situe à .70 au début des années ' 20 , les changements qu'il connaît sont à peu près les mêmes que ceux enregistrés pour la part des salaires. On peut dire que pour la période étudiée, l'évolution de la part salariale, contrôlée pour les changements de taux de salariat, a été de stable à décroissante. Dans l'hypothèse où le taux de salariat inclut les aidesfamiliaux, le revenu moyen des salariés évolue parallèlement à celui des non-salariés, alors que lorsqu'ils sont exclus, il se produit une baisse. Notons que cette baisse n'est pas due à l'inclusion des revenus de hauts salariés dans la masse salariale puisque précisément leur inclusion a pour effet d'augmenter la masse salariale.

Tableau 1

Parité salariale

$\begin{array}{cccc}\text { Années } & \text { Part salariale } & \text { Taux de salariat } & \begin{array}{c}\text { Colonne (1) divisée } \\ \text { par colonne (2) }\end{array} \\ & \text { (1) } & \text { (2) } & \text { (3) } \\ 1921 & .55 & .61 & 0.90 \\ 1978 & .73 & .89 & 0.82\end{array}$

\section{LES CHANGEMENTS STRUCTURELS ET LEURS EFFETS SUR LA PART SALARIALE}

Nous avons mentionné précédemment que la structure industrielle influait sur le niveau de la part salariale. Depuis le début du siècle, des changements importants se sont produits dans l'importance relative des divers secteurs industriels et commerciaux. Le déclin de l'industrie agricole où les taux de rémunération et la part salariale sont peu élevés par rapport à la plupart des autres industries, est un facteur qui favorise la hausse de la part salariale, pour ne donner que cet exemple. 
Pour connaître l'influence des changements structurels sur le niveau de la part salariale, il suffit de se demander quel serait le niveau de la part salariale si la pondération des secteurs économiques était demeurée la même ou encore obtenir pour chaque secteur son changement de pondération et multiplier chacun de ces changements par la part salariale dans le secteur correspondant. Ce qui donne pour chaque secteur la contribution d'origine structurelle à la hausse de la part du travail. La somme pour tous les secteurs donne cette partie de la hausse de la part salariale qui est due à des changements structurels.

Les statistiques que nous possédons avant 1926 ne sont pas suffisamment détaillées pour nous permettre de faire une étude approfondie pour la période 1910-1926. Cependant à partir de 1926, nous possédons des statistiques du revenu domestique par grands secteurs industriels. Une étude systématique faite pour une première période, soit de 1926 à 1960 nous permet de conclure que les changements. structurels de l'économie expliquent $80 \%$ de changements de la hausse de dix points de la part salariale dans le revenu domestique durant cette période. En particulier, l'importance accrue du secteur manufacturier et de celui de la construction où la masse salariale est proportionnellement plus élevée que dans les autres secteurs, contribue pour $50 \%$ de la hausse observée de la part salariale. Le développement du secteur public explique pratiquement le reste de la hausse.

Cependant, pour l'ensemble de la période d'après-guerre, soit de 1945 à 1978 , on ne peut expliquer par les changements structurels la hausse de la part salariale. En fait, $68 \%$ de la hausse de la part salariale globale est due à l'augmentation des parts salariales dans chaque secteur. Durant cette période, le secteur de la fabrication en particulier a perdu en importance mais la part salariale dans ce secteur augmente. Les changements structurels qui expliquent le reste de la hausse de la part salariale se résolvent dans la hausse de l'importance des secteurs de la construction et des finances et assurances.

On peut donc dire que durant la période d'après-guerre le facteur dominant de la hausse de la part salariale a été la hausse des masses salariales relatives à l'intérieur des principaux secteurs de l'économie, comme celui du secteur de la fabrication. Les changements structurels tombent au second plan. En fait, lorsqu'on compare les composantes de la part salariale globale entre 1946 et différentes années durant la décennie 1970, on observe que non seulement ce phénomène est important pour l'ensemble de la période étudiée, mais qu'il s'accentue à partir de $1974^{8}$.

8 BOURY SY, Aly, Part des salaires dans le revenu national; cas du Canada d'aprèsguerre (1945-1978), Université de Montréal (miméo.). 
Cette analyse globale que nous avons faite nous permet de connaître uniquement le profil des fluctuations des parts salariales des secteurs, c'està-dire ce qui reste après qu'une partie des variations se soit neutralisée par des mouvements inverses entre secteurs. En effet, la facture des variations des parts salariales sectorielles peut être telle que les fluctuations se neutralisent entre elles de sorte qu'au niveau agrégé les changements sont réduits. Il peut arriver aussi que les variations des parts salariales des secteurs soient compensées par des variations opposées de la pondération même des secteurs. Nous avons observé un tel cas dans le secteur manufacturier pour l'après-guerre où la part des revenus attribués aux salariés a augmenté, mais la baisse en importance de ce secteur a non seulement neutralisé cet effet, mais réduit la hausse même de la part salariale globale.

Nous avons voulu connaître par une étude systématique les fluctuations des secteurs et des parts salariales sectorielles, s'il ne s'agissait que d'un cas isolé. Il est en effet possible, à partir des données sectorielles, de calculer un coefficient de variation (variance) dans l'hypothèse d'interdépendance nulle, c'est-à-dire dans le cas hypothétique où les variations des secteurs ne sont pas reliées entre elles de façon systématique ${ }^{9}$. Ensuite, nous comparons ces résultats avec ceux observés et pour la part salariale courante et pour celle où la pondération des secteurs est fixe.

En d'autres mots, nous avons d'abord calculé à travers les années une variance théorique des fluctuations des parts salariales sectorielles ${ }^{10}$, ensuite, nous avons procédé à la comparaison avec la variance estimée dans deux situations. D'abord, la variance de la part salariale à pondération fixe de secteurs. Nous retenons cette dernière car, comme nous l'avons déjà mentionné, dans la réalité les poids des secteurs varient aussi bien que les parts salariales sectorielles ${ }^{11}$. Pour isoler les fluctuations des parts salariales sectorielles, il nous faut alors éliminer les changements de pondération des secteurs. Car si les fluctuations de ceux-ci sont, par exemple, correliées négativement avec les fluctuations des parts salariales sectorielles, ils contribuent à la stabilité de la part salariale macroéconomique. Mais nous voulons d'abord savoir si la fluctuation des parts salariales sectorielles se compensent entre elles, indépendamment des variations des secteurs eux-mêmes.

9 SOLOW, R.M., "A Skeptical Note on the Constancy of Relative Shares", American Economic Review, Vol. 48, septembre 1958. Pour une application voir Gérald MARION, «La part salariale dans le revenu national: L'expérience canadienne», op. cit.

10 MARION, Gérald et Aly BOURY SY, La part salariale dans le revenu national, Cahier 8114, Département de sciences économiques, Université de Montréal, 1981, À paraître dans L'Actualité Économique.

11 Tiré essentiellement de Gérald MARION et Aly BOURY SY, op. cit. Pour le détail des calculs voir cette publication. 
Si on calcule selon la méthode classique sur la période des trente-quatre années, la variance de la part salariale macroéconomique à pondération fixe, on observe qu'elle est supérieure à la variance théorique calculée sous l'hypothèse où il n'y a pas d'effets compensateurs. Ce qui signifie que les corrélations positives dans les fluctuations semblent l'emporter sur les fluctuations négatives ou compensatrices. Il n'existe pas de façon systématique de variations compensatrices dans les changements temporels des parts salariales sectorielles, car pour que cela se produise, la variance de la part salariale macroéconomique (pondération fixe) devrait être inférieure et non supérieure à la variance théorique. Ainsi la part salariale macroéconomique à pondération fixe n'est pas plus stable que le laissent supposer les fluctuations dans les secteurs industriels.

Il est intéressant de comparer cette situation avec les fluctuations à travers les années de la part salariale courante, celle où on laisse la pondération des secteurs varier selon leur importance réelle. On pourra cette fois-ci par comparaison avec les résultats précédents et avec la variance de la part salariale théorique isoler les effets des changements de poids sur les fluctuations compensatrices. Dans ce cas-ci, la différence entre la variance théorique et la variance des parts salariales courantes est importante. D'ailleurs, ces résultats révèlent que ce ne sont pas les variations compensatrices qui sont importantes. Au contraire, les fluctuations des parts salariales et la pondération des secteurs s'additionnent. En effet, le sens de l'écart, coefficient plus élevé pour la variance de la part salariale courante que pour la variance théorique, signifie que les changements de pondération vont dans le même sens que les changements dans les parts salariales sectorielles, les fluctuations s'additionnant. C'est l'interprétation que l'on doit donner au fait que c'est lorsqu'on relâche l'hypothèse de pondération fixe que la variance est la plus élevée. La corrélation n'est donc pas tellement entre les variations des parts salariales sectorielles, mais entre celles-ci et les variations de pondération des secteurs. De plus, cette corrélation est positive et non négative comme c'est le cas lorsque les fluctuations se compensent.

Bien que l'on observe dans les tableaux détaillés de nombreux cas de variations compensatrices dans la pondération des secteurs et l'évolution de la part salariale dans ces secteurs, il demeure qu'il n'y a pas de façon systématique de variations compensatrices soit dans les variations des parts sectorielles entre elles, soit dans la variation de l'importance des secteurs et de leur part salariale. En d'autres termes, l'évolution de la part du travail que nous avons observée reflète la facture des changements au niveau sectoriel. 


\section{PART DES SALAIRES ET PART DU TRA VAIL}

Derrière les variations de long terme de la part salariale, on trouve les changements qui se sont produits chez les facteurs de production, notamment dans les quantités relatives de capital et de travail. Des observations sur diverses périodes depuis 1925 , nous amènent à la conclusion que le stock de capital a crû à un rythme deux fois supérieur à celui du travail. L'évolution de la part du travail dépend de l'élasticité des parts distributives par rapport aux changements dans les quantités relatives de capital et de travail. Ce coefficient dépend lui-même de l'élasticité de substitution entre le capital et le travail de telle manière que si l'élasticité de substitution est égale à l'unité, les parts distributives demeurent stables. Cela est manifeste du fait que dans une telle situation le rapport des quantités évolue de façon inverse à l'évolution des prix relatifs du capital et du travail.

De telles variations compensatrices se sont produites au Canada et ont conduit à une certaine stabilité de la part du travail. Nous ne reprenons pas ces études détaillées ici, mais remarquons que ce genre d'analyse s'applique à l'étude de la part du travail et non des salaires.

En effet, l'analyse marginaliste est fondée sur une répartition binaire du revenu national, alors que l'évolution de la part des salaires tient compte, en plus du travail et du capital, des facteurs représentés par les «indépendants» et les entreprises non-incorporées. Comment passe-t-on de la part salariale à la part du travail et concilie-t-on ces deux concepts?

D'abord, rappelons que la part salariale comme telle a augmenté durant les derniers trois quarts de siècle. L'augmentation est significative ayant été de quelques trente points. Néanmoins, nous avons vu que cette augmentation s'accompagne de changements structurels importants. Ce qui nous amène à introduire un autre concept qui correspond davantage à la stabilité dont on vient de faire état, soit celui de la part du travail. Nous avons vu en effet que la baisse de l'importance de la catégorie des facteurs mixtes signifie que les facteurs primaires, capital et travail, entrent graduellement dans leur catégorie respective, ce qui a pour effet, entre autres, d'augmenter la part des salaires. Mais si, pour toute la période, nous nous en tenons à une répartition binaire du revenu national en imputant au travail et au capital leur revenu respectif provenant de la catégorie des facteurs mixtes, les changements de statut des entreprises non incorporées n'influent plus sur la répartition des revenus. L'agrégat que l'on obtient du côté du travail, n'est plus la masse salariale, mais les revenus du travail. La part du travail est en quelque sorte indépendante du statut des entreprises non incorporées. À la différence de la part salariale qui elle augmente tout au long 
de la période, la part du travail demeure stable pour au moins jusqu'en 1960. Quoique les auteurs ne soient pas toujours précis, il semble bien que c'est à cette répartition binaire qu'ils se réfèrent lorsque dans la théorie marginaliste ils parlent de stabilité des parts distributives.

Des études montrent que dans la catégorie mixte, la rémunération du travail et du capital est inférieure à la rémunération des facteurs de production dans leur catégorie respective ${ }^{12}$. Il y a donc une pression qui s'exerce pour une organisation de la production fondée sur la répartition binaire. Mais, si la hausse du taux de salariat est rapide, on pourra alors s'attendre à ce qu'il se produise une baisse relative, par rapport aux non-salariés, du revenu moyen des salariés. C'est en fait ce que nous avons observé. Bien que nous ayions observé une hausse de la part salariale, cette hausse se traduit par une baisse du revenu relatif moyen lorsque nous tenons compte de l'augmentation de la proportion de salariés dans la population active. En d'autres mots, la hausse du prix du travail, soumise aux pressions de l'excès d'offre parvenant des indépendants, ne compense pas l'augmentation du stock de capital possédé par les non-salariés.

\section{The Wage Share in Canada, 1910-1980}

Since 1910 there has been in Canada an increase of thirty percentage-points in the wage share in National Income. The importance of structural changes in explaining the wage share increase over time is studied. It appears that shifts in the relative importance of industrial, farm and government sectors would explain about $60 \%$ of the increase in the wage share between 1910 and 1960 . However, when only the period following world war II is considered, the results are different. It is the increase of wage share in various sectors that explains the rise in the total wage share. Finally, in the last part of the article, the author discusses the relationship between the wage share and the labour share. The labour share concept differs from the wage share in that labor income of unincorporated businesses is added to the wage bill. In view of the fact that unincorporated business sector has been decreasing since the beginning of the century, the labour share in national income will rise less than the wage share. Indeed, this aggregate tends to be more stable over the years.

12 Nous avons, en effet, pour estimer un coefficient de partage des revenus mixtes, entre le capital et le travail, calculé le revenu du travail et du capital en supposant qu'ils aient la même rémunération pratiquée pour le travail et le capital. Les résultats donnent des chiffres nettement gonflés par rapport aux revenus mixtes observés. 